\title{
Adaptive redundant message deletion mechanism for opportunistic network
}

\author{
Xie $\mathrm{Yi}^{1, \mathrm{a}}$, Pan $\mathrm{Ke}^{1, \mathrm{~b}}$, Lu Jinping ${ }^{1, \mathrm{c}}$ and Li Wuyou ${ }^{2, \mathrm{~d}}$ \\ ${ }^{1}$ Chongqing Institute of Telecommunications, Chongqing, 401336, China \\ ${ }^{2}$ Chongqing University of Posts and Telecommunications, Chongqing, 400065, China \\ a81350378@qq.com, bpanke@chinattl.com, clujinping@chinattl.com, d835698683@qq.com
}

Keywords: opportunistic network, redundant message deletion, acknowledge message, message density.

\begin{abstract}
The flooding method is utilized to forward the acknowledge message to delete the redundant copies of message. As a result, lots of network resources will be consumed by this kind of operation. A novel message deletion mechanism is proposed in this paper. Taking into account the hop count of ACK message, historical forwarding status and regional message density, the ACK message is forwarded to the nodes in the network with the distributed manner to remove the redundant copies of message reasonably. Simulation results show that the redundant copies of message can be deleted with less ACK message by the proposed mechanism; moreover, the network performance can be improved obviously.
\end{abstract}

\section{Introduction}

The data transmission in opportunistic network[1] is accomplished in a cooperative manner. Routing in opportunistic network adopts a store-carry-and-forward scheme by which messages are forwarded through intermediate nodes leveraging opportunistic encountering.

Current data forwarding mechanism commonly used approach with a copy of the flooding control to minimize the cost of network resources and maximize the carrying capacity of the network[2-3]. The defects of this mechanism is obviously, that is, multiple copies will consume more network bandwidth and storage resources.

At present, the methods to remove the redundancy messages mainly by distributing an acknowledgment(ACK) message. Researchers proposed a passive ACK message distribution method[4], node carrying ACK message does not perform active diffusion process, but when there are other nodes forward the message which has been successfully delivered, the corresponding acknowledgment message forwarding to each other to remove redundant messages. However, ACK information diffusion in the network is slow, it can't promptly remove redundant copy of the message. Literature[5] proposed a active distribution method, each node holds the corresponding ACK list to identify which ACK message carried. After the encounter with other nodes, they're forwarding all ACK message with each other, which quickly spread ACK message. Obviously, in the case of large-scale network or generated more messages, forward messages ACK consuming network resources will increase rapidly. In addition, if the encounter interval between two nodes is shorter, forwarding all ACK message will be consume unnecessary overhead.

An adaptive ACK distribution mechanism(AAD) is proposed in this paper, Taking into account the hop count of ACK message, historical forwarding status and regional message density, the ACK message is forwarded to the nodes in the network with the distributed manner. In consideration of the difference necessity of nodes for ACK message, ACK distribution mechanism proposed to better use of network resources. 


\section{ACK Message}

For any node, when a node as the destination received message, it will generates ACK message to indicate the message has been successfully delivered. The ACK message in opportunistic network is not to achieve reliable data transmission but to delete redundant messages, which prevent to the transmission and increase resource utilization rate.

In the ACK list maintained in node, each record corresponds to a ACK message. It's structure as shown in Fig. 1. The source ID and the destination ID identify the message's source node and destination node. Message ID identifies the message. Survival time records the ACK message to live the longest time in the network, the node periodically check ACK list, when the record of the ACK message survival time expires, then delete the record.

\begin{tabular}{|c|c|c|c|}
\hline Source ID & Destination ID & Message ID & TTL \\
\hline
\end{tabular}

Fig.1 Structure of ACK message

\section{Adaptive Distribution Mechanism of ACK}

Each node carry different message, the demand for the ACK message is also different. At the same time, ACK message needs to forward the node carried the redundancy message and the node may receive the redundancy pointedly. In this paper, the adaptive ACK distribution mechanism includes two stages, flooding phase and forward control phase.

(1) Flood phase: In order to delete redundant messages with the quickest way, ACK message carrying node forward information to other nodes in the form of flooding. After the encounter between nodes, forwarding all ACK message carried to each other. In order to effectively limit the consumption of resources generated by the forwarding process, we use the number of hops-based approach to control the ACK forward range. Each ACK message having a corresponding forwarding limit, indicating that the maximum number of message forwarding. Fig. 2 describe the history forwarding records when upper limit of $L=3$.

(2) Forward control phase: when the upper limit of the forwarding of an ACK message is turn to 0 , entering the forward control phase. At this stage, regional message density decide the forwarding process of ACK message. If the density of message $\mathrm{i}$ is high, it indicates that less ACK message within the region corresponding to the message $i$. At this time the message $i$ will forwards in the area with high probability and infect remaining nodes, in which case need to transfer ACK message to reduce the redundancy of message $i$, it effectively reduce the waste of resources brought by redundant messages.

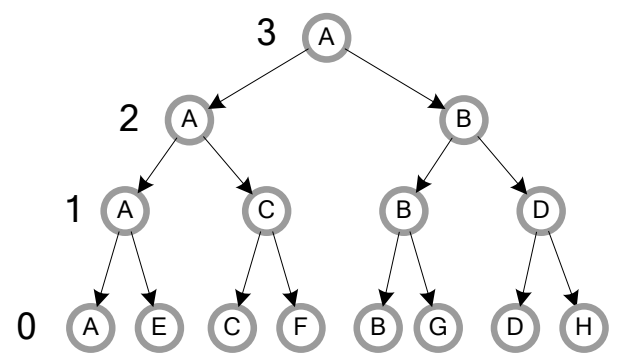

Fig 2. Illustration of hop-based forwarding

Nodes need to maintain a history information table when they encounter. As shown in Fig.3, including the address of encounter node, encounter time and the message ID carried by node. Regional message density obtained in the estimation of the list's information.

\begin{tabular}{|c|c|c|}
\hline Address & Encounter Time & Message Id List \\
\hline
\end{tabular}

Fig.3 Historical meeting information of node

Based on encounter node number $N(T)$ within the time $T$ and the node number $M_{i}(T)$ which carry the message $i$, the regional message density $M D_{i}(T)$ of node $i$ defined as follows: 


$$
M D_{i}(T)=\frac{M_{i}(T)}{N(T)}
$$

According to the the regional message density of node $\mathrm{i}$, node performs the decision of ACK message forwarding according to formulate (2). $\rho_{t h}$ is the regional density threshold.

$$
P_{\text {forward }}=\left\{\begin{array}{l}
0, M D_{i}(T) \leq \rho_{\text {th }} \\
1, M D_{i}(T)>\rho_{\text {th }}
\end{array}\right.
$$

Adaptive ACK distribution mechanism is shown in Fig. 4,which $\mathrm{Y}$ is encounter node, ACK_i for the ACK message of message $i$.

After adding ACK routing, the step will be different. For a node X, Y exemplified, X broadcasts Hello packets with local message list periodically to find the neighbor nodes. When $\mathrm{Y}$ receives a Hello packet sent by node $\mathrm{X}$, return a reply to request the required message and forward the ACK message determined by Fig.4. After receiving the Reply, X delete the redundant message according to the received ACK list, and then forward request packet follow the forwarding mechanism.

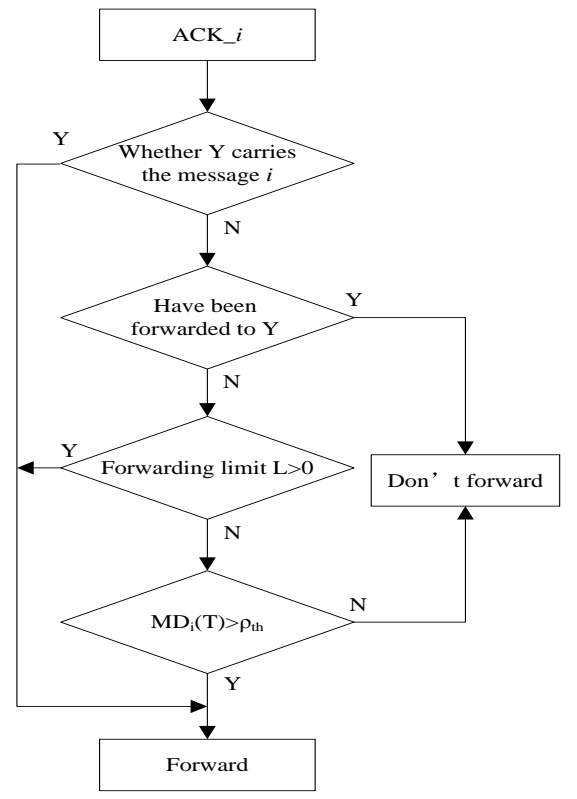

Fig.4 Adaptive forwarding mechanism of ACK

\section{Numerical Analysis}

The Opportunistic Network Environment(ONE)[6] is employed to verify the validity of the proposed mechanism ADD. The comparison is done with Epidemic and PROPHT. We compare our proposed scheme with Flooding ACK and NO-ACK under different cache size and different number of nodes. Simulation scenario contains three types of nodes: tram, cars and pedestrians. The forwarding limit is $L=6$, other relevant parameters is shown in Table 1.

Table 1 Simulations parameters

\begin{tabular}{cc}
\hline Paramter & Value \\
\hline Simulation time $/ \mathrm{h}$ & 12 \\
Simulation area $/ \mathrm{m} \times \mathrm{m}$ & $4300 \times 3400$ \\
Node Group & $6(2$ pedestrians; 1 cars; 3 trams $)$ \\
Number of Node & pedestrians and cars(40-80/Group); trams(2/Group) \\
Moving speed $/ \mathrm{km} \cdot \mathrm{h}^{-1}$ & pedestrians(1.8-5.4); cars(10-50); tram(25-36) \\
Buffer size $/ \mathrm{MByte}$ & Pedestrians and cars(10-50); trams(50) \\
Transmission range $/ \mathrm{m}$ & 10 \\
Size of message $/ \mathrm{KByte}$ & $500-1000$ \\
\hline
\end{tabular}




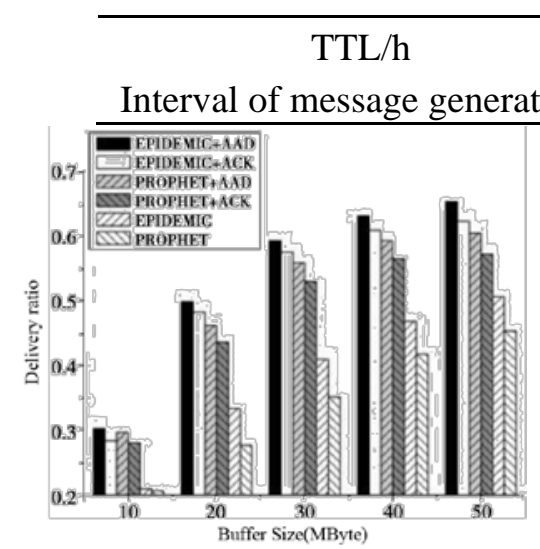

(a)

Fig.5 Network performance under different buffer size

5

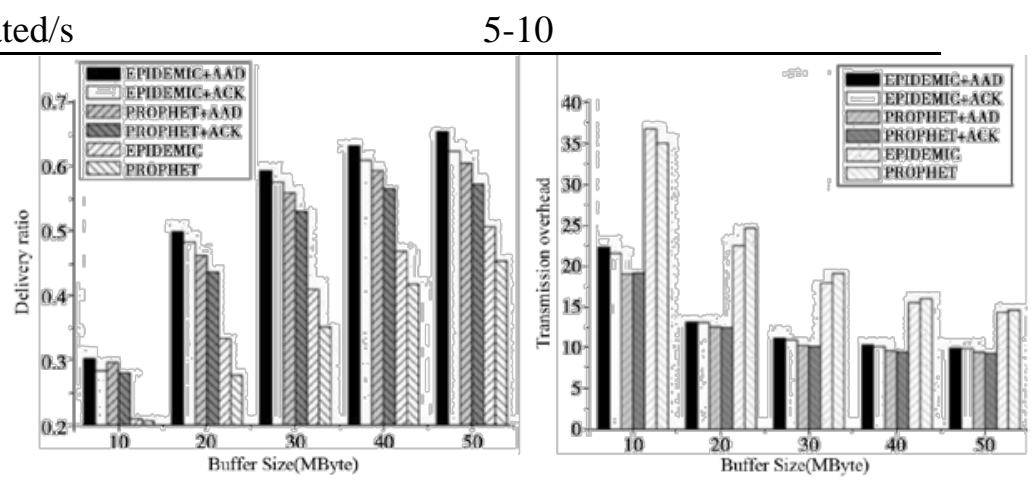

(c)

Figure 5 depicts the trend of the network performance under different cache size. As seen in Fig. 5a, After using AAD routing can be less ACK message to achieve the goal of eliminating redundant messages in improving delivery rates while reducing the average delay of messages. Delivery delay gradually increased in Fig. 5b, this is because the message due to insufficient buffer size is deleted. Fig. 5c showed that AAD and flooding almost have same overhead, indicate AAD can be achieved same network performance compared with flooding.

\section{Summary}

This paper studies the role ACK message on the redundant messages deleted and clarified the problems caused by flooding ACK message. Based on the need for the different ACK message for node, we proposed ACK adaptive distribution mechanism, Simulation results show that, ACK distribution mechanism can effectively improve the delivery rate, lower latency and improve network performance.

\section{Acknowledgement}

This work was supported by Chongqing Natural Science Foundation (Grant No. cstc2013jjB40006), and Chongqing Science and Technology Personnel Training Program (Grant No. cstc2013kjrc-qnrc40007).

\section{References}

[1] Xiong Y P, Sun L M, Niu J W, et al. opportunistic networks[J]. Journal of Software, 2009, 20(1): 124-137

[2] Wu D P, Lou P W, Liu Q S, et al. Coding redundancy controlled data forwarding mechanism in opportunistic networks[J]. Journal on Communications, 2015, 36(3): 37-48.

[3] Wu D P, Zhou J E, Wang R Y, et al. Message-redundancy Estimating Adaptive Buffer Management Mechansim for Opportunistic Network[J]. Journal of Electronics and Information Technology, 2012, 34(1): 101-107.

[4] Harras K A, Almeroth K C, Belding-Royer E M. Delay tolerant mobile networks (DTMNs): Controlled flooding in sparse mobile networks[M]// NETWORKING 2005. Networking Technologies, Services, and Protocols; Performance of Computer and Communication Networks; Mobile and Wireless Communications Systems. Springer Berlin Heidelberg, 2005: 1180-1192.

[5] Mundur P, Seligman M, and Lee G. Epidemic routing with immunity in delay tolerant networks[C]// Military Communications Conference. San Diego, America: IEEE Press. 2008: 1-7. 
[6] Keranen A, Ott J, Karkkainen T. The ONE simulator for DTN protocol evaluation[C]// Proceedings of the 2nd International Conference on Simulation Tools and Techniques. Rome, Italy: ACM Press, 2009: 1-10. 